\title{
Radial Density Profiles of Fluorine Negative Ions and Electrons around a Magnetized String Plasma in $\mathrm{CF}_{4}$ Gas
}

\author{
M Abid IMTIAZ and Tetsu MIENO*
}

Department of Physics, Faculty of Science, Shizuoka University, Ooya, Suruga-ku, Shizuoka 422-8529, Japan

Radial density profiles of electrons and fluorine negative ions around a stringtype $\mathrm{CF}_{4}$ plasma across a magnetic field are measured using a wire-type Langmuir probe. Modified Bohm theory is applied to evaluate negative-ion density, and it is confirmed that negative ions are well confined between two end plates. Parameter dependences of the density profiles are also measured. Simple density models for electrons and negative ions are proposed, and their radial density profiles are obtained by solving the equations of continuity. The experimental and theoretical results are in good agreement for electrons, whereas there are some discrepancies for negative ions. An unexpected small recombination rate coefficient of negative ions and radial electric field might be the reason for this discrepancy.

KEYWORDS: fluorine negative ion, ion source, string-type plasma, radial diffusion, Langmuir probe, magnetized plasma, plasma etching, carbon tetrafluoride

\footnotetext{
* E-mail address: sptmien@ipc.shizuoka.ac.jp
} 


\section{Introduction}

Ultra-fine etching using electronegative plasmas is a likely candidate to improve the plasma etching process for the production of higher quality ultra-largescale integrations (ULSIs). Plasma etching with negative ions has been shown to be effective to reduce ${ }^{1,2)}$ the damage ${ }^{3-6)}$ that eventually occurs during conventional plasma etching. Therefore, clear knowledge about the production, diffusion, and extinction characteristics of negative ions, as well as positive ions and electrons, produced by electronegative gas discharge is vital to ensure efficient industrial application of negative-ion plasmas.

A column-type magnetized plasma with electronegative gas produces negative ions, where negative ions accumulate around the plasma column ${ }^{7)}$ because they are confined sufficiently along the magnetic field. Using this property, column-type negative ion sources have been developed. ${ }^{8,9)}$ Separative diffusion of fluorine negative ions is realized around a multistring-type plasma made by a multihole-type obstacle plate, and a large-diameter negative-ion source can be produced. ${ }^{10)}$

In this study, in order to clarify the process of accumulation of negative ions and to improve negative-ion density, careful measurement of negative-ion density $\left(\mathrm{F}^{-}\right)$ and electron density around a simple string-type plasma is carried out. The radial density profiles of negative ions and electrons under different gas pressures and magnetic fields are measured. Theoretical models of the production, diffusion, and extinction of electrons and $\mathrm{F}^{-}$ions in the magnetized $\mathrm{CF}_{4}$ plasma are proposed. 
Calculations using the continuity equations of electrons and negative ions according to the model have been carried out to know whether the models support the experimental results.

\section{Theory}

The proposed model of a string-type magnetized plasma is shown in Fig. 1, where production, diffusion, and extinction characteristics of fluorine negative ions $\left(\mathrm{F}^{-}\right)$produced by a column-type magnetized plasma with $\mathrm{CF}_{4}$ gas have been investigated. Initially the plasma is produced by electron-beam injection along the magnetic field. In this model, the dominant charged species are electrons, $\mathrm{F}^{-}$ions and $\mathrm{CF}_{3}^{+}$ions, and the later two species are produced by dissociative attachment $(\mathrm{e}+$ $\left.\mathrm{CF}_{4} \rightarrow \mathrm{CF}_{3}^{+}+\mathrm{F}^{-}\right)$and electron impact ionization $\left(\mathrm{e}+\mathrm{CF}_{4} \rightarrow \mathrm{CF}_{3}^{+}+\mathrm{F}+2 \mathrm{e}\right)$, respectively. The $\mathrm{F}^{-}$ions produced diffuse radially and accumulate around the plasma column. In addition to the experimental investigation, calculations using continuity equations for $\mathrm{F}^{-}$ions and electrons, as they appear in the proposed model, have been carried out.

In the DC-discharge plasma, thermal electrons in the plasma are produced by injecting an electron beam along the magnetic field with $100 \mathrm{eV}$ of energy. Some of the thermal electrons are lost at the end electrodes after diffusing along the magnetic filed or by attaching to $\mathrm{CF}_{4}$ molecules. On the other hand, the negative ions produced are well-confined along the magnetic field by sheath potentials at the end plates and are partially lost by recombining with positive ions. Around the 
string-type plasma, the radial-diffusion speeds of electrons and negative ions are very different, which produces the ion-ion plasma. Moreover, the bipolar-type diffusion in the magnetic field establishes a substantial radial electric field such that the radial diffusions of negative ions are decelerated.

\subsection{The model equations}

We consider the proposed model illustrated in Fig. 1. Taking into account the perpendicular mobility $\left(\mu_{\perp}\right)$ of the species and the radial electric field $\left(E_{\perp}\right)$, the continuity equations for electrons and negative ions can be written as

$$
\begin{aligned}
& K_{\mathrm{iz}} n_{\mathrm{EB}} n_{0}+D_{\perp e} \nabla^{2} n_{e}+\mu_{\perp e} \nabla \cdot\left(n_{e} E_{\perp}\right)-\alpha n_{e}=0, \\
& K_{\mathrm{att}} n_{e} n_{0}+D_{\perp-} \nabla^{2} n_{-}+\mu_{\perp-} \nabla \cdot\left(n_{-} E_{\perp}\right)-K_{\mathrm{rec}} n_{+} n_{-}=0,
\end{aligned}
$$

respectively. In these equations, $K_{\mathrm{iz}}, K_{\mathrm{att}}$, and $K_{\mathrm{rec}}$ symbolize the ionization rate constant of the electron beam, dissociative attachment coefficient of $\mathrm{F}^{-}$ion production, and ion-ion recombination coefficient, respectively. $D_{\perp e}$ and $D_{\perp-}$ are the radial diffusion coefficients for electrons and negative ions, respectively. $n_{e}$ and $n_{-}$are the densities in the bulk plasma region of electrons and negative ions, respectively. $n_{\mathrm{EB}}$ and $n_{0}$ are the beam electron density and neutral molecule density, respectively. A constant, $\alpha$, is introduced in this model to show an averaged electron absorption coefficient in eq. (1), which includes both the time-averaged absorption probability at the end plates and the probability of electron-ion attachment. The equations of continuity are solved in a cylindrical coordinate system using MATHEMATICA 
version 5.2 (Wolfram Research) software adopting adequate boundary conditions.

\subsection{Solution to the continuity equations}

In order to solve the continuity equation for electrons [eq. (1)], it is assumed that thermal electrons are mainly produced by the collisions of beam electrons with the neutrals within the electron-beam diameter region $\left(\varnothing_{\mathrm{EB}}=10 \mathrm{~mm}\right)$ and are lost by axial diffusion and absorption at the ends. Therefore, in the region outside the beam diameter, $r \geq 0.005 \mathrm{~m}$, production of electrons can be neglected, i.e., the first term in eq. (1) can be taken as equal to zero. At the periphery of the electron beam, electron density is assumed from the experimental measurement to be $\left.n_{e}\right|_{0.005}=3 \times 10^{15} \mathrm{~m}^{-3}$. Moreover, the gradient of electron density at the inner walls of the vessel (at $r=0.1 \mathrm{~m}$ from the axial center) is considered to be zero, i.e., $\partial n_{e} /\left.\partial r\right|_{0.1}=0 . \quad D_{\perp e} \approx 0.04 \mathrm{~m}^{2} \mathrm{~s}^{-1}$ is used for a typical value of an applied magnetic field of $0.03 \mathrm{~T}^{11)}$ and an electron temperature of $2 \mathrm{eV}$. The probability of absorption of electrons is assumed to be $\alpha=2.0 \times 10^{3} \mathrm{~s}^{-1}$ from the experimental results.

For negative ions, it is assumed that $\mathrm{F}^{-}$ions are produced mainly by electron attachment processes within the electron beam diameter region and are diffused radially and lost by recombination with positive ions. Therefore, outside the electron beam region, production of $\mathrm{F}^{-}$ions can be neglected. This assumption leads to the first term in eq. (2) at $r \geq 0.005 \mathrm{~m}$ being set as zero. At the edge of the beam, the negative-ion density and gradient of negative-ion density are taken to be $\left.n_{-}\right|_{0.005}=3 \times 10^{16} \mathrm{~m}^{-3}$ and $\partial n_{-} /\left.\partial r\right|_{0.005}=-6.3 \times 10^{18} \mathrm{~m}^{-4}$, respectively, according to the 
experiment. The perpendicular diffusion coefficient of negative ions $D_{\perp-} \approx 0.5 \mathrm{~m}^{2} \mathrm{~s}^{-1}$ for an applied magnetic field of $0.03 \mathrm{~T}$ and a negative-ion temperature $T_{-}=0.1 \mathrm{eV}$ is

used. ${ }^{12,13)}$ The recombination rate coefficient of negative ions with positive ions is set according to the reference, $K_{\mathrm{rec}}=5 \times 10^{-13} \mathrm{~m}^{3} \mathrm{~s}^{-1} .{ }^{14)}$

\section{Experimental Procedure}

An illustration of the experimental setup is shown in Fig. 2. A $2.0 \mathrm{~m}$ long vacuum vessel $21 \mathrm{~cm}$ in diameter made of stainless steel is set across eight solenoid coils producing a magnetic field $B=0-0.15 \mathrm{~T}$ along the $z$-axis. An electron beam 10 $\mathrm{mm}$ in diameter with a beam energy $\psi_{\mathrm{BE}}=100 \mathrm{eV}$ is injected by an electron beam source placed at the axial center. An electrically grounded anode plate with a gridded orifice $10 \mathrm{~mm}$ in diameter forms the shape of the electron beam. The beam current of this source is as high as $0.05 \mathrm{~A}$. An end plate of $20 \mathrm{~cm}$ diameter at ground potential is set $77 \mathrm{~cm}$ from the beam source. Hot-wire-type Langmuir probes made of Ta wire of $0.2 \mathrm{~mm}$ diameter and shaped into half-circle of $1.5 \mathrm{~mm}$ diameter are used to diagnose the plasma. Results reported in this article are obtained using the probe placed $21 \mathrm{~cm}$ from the anode plate.

The modified Bohm method is used to evaluate negative-ion density from the Langmuir probe data of the negative-ion-rich plasma. For a weak electronegative plasma, where $n_{-} / n_{e}<2$, the normal Bohm condition can be used at the sheath region of the plasma boundary, and $n_{-}$can be estimated from the Langmuir probe 
measurement. ${ }^{7)}$ On the other hand, in the presence of many negative ions $\left(n_{-} / n_{e} \geq 2\right)$, the magnitude of presheath potential $\Delta \emptyset_{p s}$ is reduced, and this potential reflects a quantity of negative ions in the presheath region. In this article, this condition is called "the modified Bohm condition". In this condition, the ion saturation current density flowing to the probe is given by ${ }^{8,15)}$

$$
J_{i s} \approx e\left[n_{e b} \exp \left(-\frac{e \Delta \emptyset_{p s}}{\kappa T_{e b}}\right)+n_{-b} \exp \left(-\frac{e \gamma \Delta \emptyset_{p s}}{\kappa T_{e b}}\right)\right] \times \sqrt{\frac{2 e \Delta \emptyset_{p s}}{M_{+}}},
$$

where $n_{e b}$ and $n_{-b}$ are the electron density and negative-ion density in the bulk plasma, respectively, and $\gamma=T_{e b} / T_{-b}$ (the ratio of electron temperature to negative-ion temperature in the bulk plasma). ${ }^{8)}$

According to the calculation method described in ref. 7, we can evaluate $n_{-b} / n_{e b}$ from the probe data, where the charge neutrality condition in the bulk plasma, $n_{e b}+n_{-b}=n_{i b}\left(n_{i b}\right.$ : positive-ion density in bulk plasma) is used. Probe data at a reference point are used where negative-ion density is negligible and charge neutrality, $n_{e 0}=n_{i 0}\left(n_{e 0}\right.$ and $n_{i 0}$ : electron density and positive-ion density at the reference point, respectively) is satisfied. Then, eq. (3) is modified to be

$$
\frac{n_{-b}}{n_{e b}} \approx \frac{J_{i s} / n_{e b}}{J_{i s 0} / n_{e 0}} \exp \left(\frac{e \gamma \Delta \emptyset_{p s}}{\kappa T_{e b}}-\frac{1}{2}\right) \sqrt{\frac{\kappa T_{e 0}}{2 e \Delta \emptyset_{p s}}}-\exp \left(\frac{e \Delta \emptyset_{p s}(\gamma-1)}{\kappa T_{e b}}\right),
$$

where $J_{i s 0}$ and $T_{e 0}$ stand for the positive ion current density and electron temperature at the reference point, respectively. The presheath potential $e \Delta \emptyset_{p s} / \kappa T_{e b}$ as a function of $n_{-b} / n_{e b}$ can be evaluated for different values of $\gamma$ from the modified Bohm method, as 
stated in equations (6.4.9) and (6.4.10) in ref. 15. Therefore, $n_{-b} / n_{e b}$ can be obtained by measuring $\left(J_{i s} / n_{e b}\right) /\left(J_{i s 0} / n_{e 0}\right), T_{e b}$, and $T_{e 0}$, and using eq. (3) and Fig. 3 of ref. 8, in which the asymptotic method in the graph is utilized.

When $e \Delta \emptyset_{p s}$ is smaller than the positive-ion temperature in the bulk plasma, $\kappa T_{+b}$, the ion saturation current to the probe is influenced by the thermal ion flow. ${ }^{8)}$ In this experiment, both $\kappa T_{+b}$ and the negative-ion temperature $\kappa T_{-b}$ are assumed to be 0.1 $\mathrm{eV}$, which is suitable for usual low-pressure laboratory plasmas, ${ }^{12,13)}$ and the effect of positive-ion temperature on the $n_{-b} / n_{e b}$ evaluation can be neglected.

\section{Results and Discussion}

Radial density profiles of negative ions and electrons from the expereiment and from the calcualtion are compared. In considering the polarity of the radial electric field, the field decelerates the negative ions and electrons, and accelerates the positive ions. However, the effect of $E_{\perp}$ on $n_{e}$ is very small. As the perpendicular mobility of electrons and $E_{\perp}$ do not have much influence on the electron density distribution, the experimental results of radial distributions of $n_{e}$ are in good agreement with the results obtained by solving the model equation for electrons as shown in Fig. 3(a).

The radial density distribution of negative ions changes owing to the effects of $E_{\perp}$, which could cause the discrepancies between the theoretical evaluation and experimental measurements of $n_{-}$shown in Fig. 3(b). With the assumption in the diffusion equation that no negative ions are produced in the bulk plasma region, the effect of production of negative ions at the beam edge region might have been 
underestimated. Moreover, the recombination coefficient cited in ref. 14 and used in the calculations may be too large to be used under the present condition, which strongly modifies the radial denisty profile. Nonetheless, in this collisional plasma, $\boldsymbol{E} \times \boldsymbol{B}$ drift and diamagnetic drift can also be important, as they would produce instabilities resulting in a greater diffusion, which would increase the density of negative ions in the bulk plasma region.

Radial distributions of $n_{e}$ and $n_{-}$, and their dependences on magnetic fields at a typical gas pressure of $0.6 \mathrm{~Pa}$ are measured and shown in Fig. 4. Both $n_{e}$ and $n_{-}$ decrease with an increase in $B$ from 0.013 to $0.15 \mathrm{~T}$. The higher magnetic field leads to a lower electron temperature and higher-density electron distribution within the beam region because electrons are confined for a longer time in the plasma and loose energy due to multiple collisions. On the other hand, the electron density increases because more ionizing collisions take place. A decrease in negative-ion density in the bulk plasma region with an increase in magnetic fields can be understood in terms of the diffusion coefficient of negative ions that decreases with increasing $B$.

Magnetic-field dependences of the density profiles of electrons and negative ions obtained by the calculations are shown in Fig. 5. As the perpendicular diffusion coefficients of the species decrease with $B$, radial profiles of $n_{e}$ and $n_{-}$tend to decrease with $B$.

Radial distributions of $n_{e}$ and $n_{-}$, and their dependences on pressures are shown in Fig. 6. In the edge plasma region, $n_{e}$ tends to increase with pressure as $T_{e}$ 
decreases, whereas in the source region $(r \approx 0-0.5 \mathrm{~cm}) n_{e}$ tends to decrease with pressure. The electron-retarding field, which is inversely proportional to $T_{e}$, increases as the pressure increases. The electron collision mean-free-path, $\lambda$, decreases with pressure, and the electron temperature decreases. This increase of collision frequency creates more thermal electrons, and hence lowers the density distribution of energetic electrons in the source region, eventually increasing the density distribution of thermal electrons in the bulk plasma. However, it is conjectured that higher collision frequencies among electrons and $\mathrm{CF}_{4}$ molecules would reduce the production rate of $\mathrm{F}^{-}$ions via dissociation of excited molecules and cause $n_{-}$to decrease with pressure.

Pressure dependences of the density profiles of electrons and negative ions were also calculated and the results are shown in Fig. 7. As the perpendicular diffusion coefficients of the species increase with $p$ (as collision frequency increases), radial profiles of $n_{e}, n_{-}$tend to increase with $p$ as shown in Fig. 7. As the pressuredependent variations are not large, the experimental results do not show strong pressure dependence of the density distributions.

The density profile of negative ions, $n_{-}$, obtained experimentally is not quite consistent with the calculated results as shown in Fig. 3(b). Therefore, calculation of negative-ion density by changing the recombination coefficient was carried out and the results are shown in Fig. 8, where $B=0.01 \mathrm{~T}$. A coefficient smaller than the value from ref. 14 tends to move to the calculated values closer to the experimental results, which suggests that the recombination rate coefficient is much smaller than the value 
presented in the reference.

The perpendicular mobilities $\mu_{\perp}$ of electrons and $\mathrm{F}^{-}$are 0.14 and $38.5 \mathrm{~m}^{2} \mathrm{~V}^{-1} \mathrm{~s}^{-1}$, respectively, as calculated for $B=0.01 \mathrm{~T}, T_{e}=1.0 \mathrm{eV}$, and $T_{-}=0.1 \mathrm{eV}$. When $E_{\perp}=$ $2.9,5.0$, and $10 \mathrm{~V} / \mathrm{m}$, the calculated results of the radial profiles of $n_{e}$ and $n_{-}$are shown in Fig. 9. The radial electric field retards negative-ion diffusion, and the average positive radial electric field of $29 \mathrm{~V} / \mathrm{m}$ in the experiment causes a negative effect on the theoretical consistency. Therefore, the diffusion caused by the radial electric field is not very important in this experiment.

\section{Conclusions}

Thermal electrons are confined axially by a magnetic field, and radial expansion is very small for $B=0.03 \mathrm{~T}$. Before radial diffusion, the electrons tend to disappear at the axial boundary. Fluorine negative ions, $\mathrm{F}^{-}$, produced in the plasma diffuse radially and are well-confined along the magnetic field, bouncing at the boundary sheath. The radial density profile of electrons is consistent with the model calculation, whereas the radial density profile of $\mathrm{F}^{-}$is not well clarified by the model calculation. By using a smaller recombination rate coeficent for $\mathrm{F}^{-}$ions, the calculated values approach the experimental results, which we suggest, is the reason of this discrepancy. The $\boldsymbol{E} \times \boldsymbol{B}$ drift caused by the radial electric fields and some instability assists the diffusion, but further study is necessary to clarify these effects. The electron-beam current, magnetic field, and gas pressure are the controlling parameters for negative-ion production. 


\section{Acknowledgements}

This work is supported by a grant-in-aid from the Ministry of Education, Culture, Sports, Science, and Technology (MEXT), Japan.

\section{References}

1) H. Shindo, Y. Sawa, and Y. Horiike: Jpn. J. Appl. Phys. 34 (1995) L925.

2) T. Shibayama, H. Shindo, and Y. Horiike: Plasma Sources Sci. Technol. 5 (1996) 254.

3) K. P. Cheung and C. P. Chang: J. Appl. Phys. 75 (1994) 4415.

4) H. Ootera, T. Oomori, M. Tuda, and K. Namba: Jpn. J. Appl. Phys. 33 (1994) 4276.

5) T. Nozawa, T. Kinoshita, T. Nishizuka, A. Narai, T. Inoue, and A. Nakaue: Jpn. J. Appl. Phys. 34 (1995) 2107.

6) T. Kinoshita, M. Hane, and J. P. McVittie: J. Vac. Sci. Technol. B 14 (1996) 560.

7) R. Kawai and T. Mieno: Jpn. J. Appl. Phys. 36 (1997) L1123.

8) T. Mieno and A. Ogawa: Jpn. J. Appl. Phys. 38 (1999) 4586.

9) N. Sato: Plasma Sources Sci. Technol. 3 (1994) 395.

10) M. Abid Imtiaz, S. Tsuruta, and T. Mieno: Plasma Sources Sci. Technol. 16 
(2007) 324 .

11) F. F. Chen: Introduction to Plasma Physics and Controlled Fusion (Plenum Press, New York, 1984) 2nd ed., p. 172.

12. T. Nakano, H. Ohtake, and S. Samukawa: Jpn. J. Appl. Phys. 35 (1996) L338.

13) H. -J. Woo, K. -S. Chung, and T. Lho: Proc. 6th Int. Conf. Reactive Plasmas, Sendai, 2006, p. 779.

14) A. Kono, M. Haverlag, D. Passchier, G. M. W. Kroesen, and F. J. deHoog: J. Appl. Phys. 70 (1991) 2939.

15) M. A. Lieberman and A. J. Lichtenberg: Principles of Plasma Discharges and Materials Processing (JohnWiley \& Sons, New York, 1994) p. 167. 


\section{Figure captions}

Fig. 1. Model figure of negative-ion accumulation around a string-type magnetized plasma. The plasma is produced by electron beam injection.

Fig. 2. Illustration of experimental setup for string-type plasma.

Fig. 3. Radial profiles of (a) $n_{e}$ and (b) $n_{-}$obtained by experiment and using model calculations. $\quad D_{\perp e}=0.04 \mathrm{~m}^{2} \mathrm{~s}^{-1}$ and $\mu_{\perp e}=0.14 \mathrm{~m}^{2} \mathrm{~V}^{-1} \mathrm{~s}^{-1} . \quad D_{\perp-}=4.7 \mathrm{~m}^{2} \mathrm{~s}^{-1}$ and $\mu_{\perp e}=38.5$ $\mathrm{m}^{2} \mathrm{~V}^{-1} \mathrm{~s}^{-1} . \quad B=0.03 \mathrm{~T}$ and $p=0.6 \mathrm{~Pa}$ are measured at $z=21 \mathrm{~cm}$ from the anode.

Fig. 4. Radial profiles of (a) $n_{e}$ and (b) $n_{-}$for four magnetic fields obtained experimentally at a pressure $p=0.6 \mathrm{~Pa} . z=21 \mathrm{~cm}$ from the anode.

Fig. 5. Magnetic field dependences of radial profiles of (a) $n_{e}$ and (b) $n_{-}$calculated using the model equations. $\quad p=0.6 \mathrm{~Pa}$.

Fig. 6. Radial profiles of (a) $n_{e}$ and (b) $n_{-}$for four gas pressures obtained experimentally in a magnetic field of $B=0.03 \mathrm{~T} . \quad z=21 \mathrm{~cm}$ from the anode.

Fig. 7. Dependence of radial profiles of (a) $n_{e}$ and (b) $n_{-}$on gas pressure calculated using the model equations. $\quad B=0.03 \mathrm{~T}$.

Fig. 8. Dependence of radial profiles of (a) $n_{-}$and (b) $n_{+}$on recombination coefficient calculated using the model equations. $D_{\perp-}=0.5 \mathrm{~m}^{2} \mathrm{~s}^{-1}$.

Fig. 9. Radial profiles of (a) $n_{e}$ and (b) $n_{-}$calculated using the model equations for $E_{\perp}=2.9,5.0$, and $10 \mathrm{Vm}^{-1} . \quad B=0.03 \mathrm{~T}, T_{e}=1.0 \mathrm{eV}$ and $T_{-}=0.1 \mathrm{eV}$ 


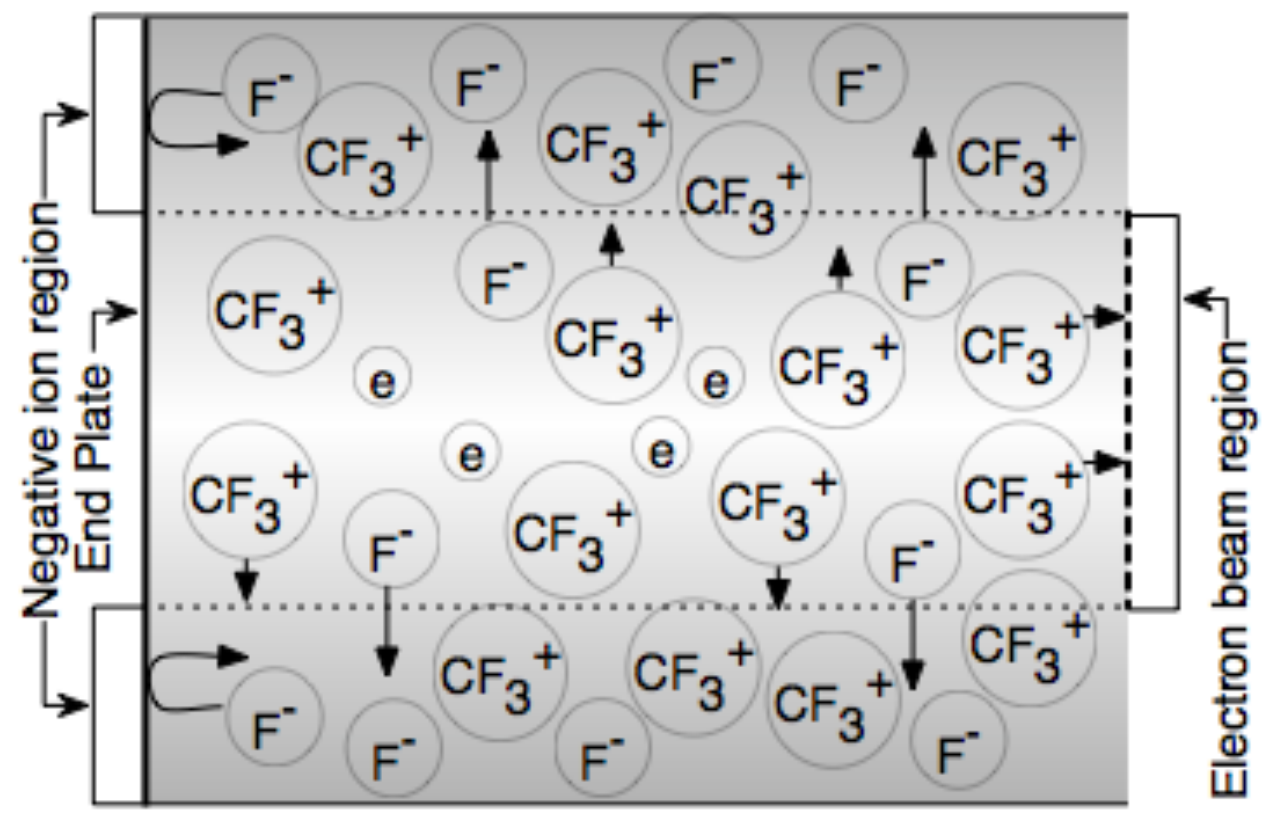

Fig. 1. Model figure of negative-ion accumulation around a string-type magnetized plasma. The plasma is produced by electron beam injection. 


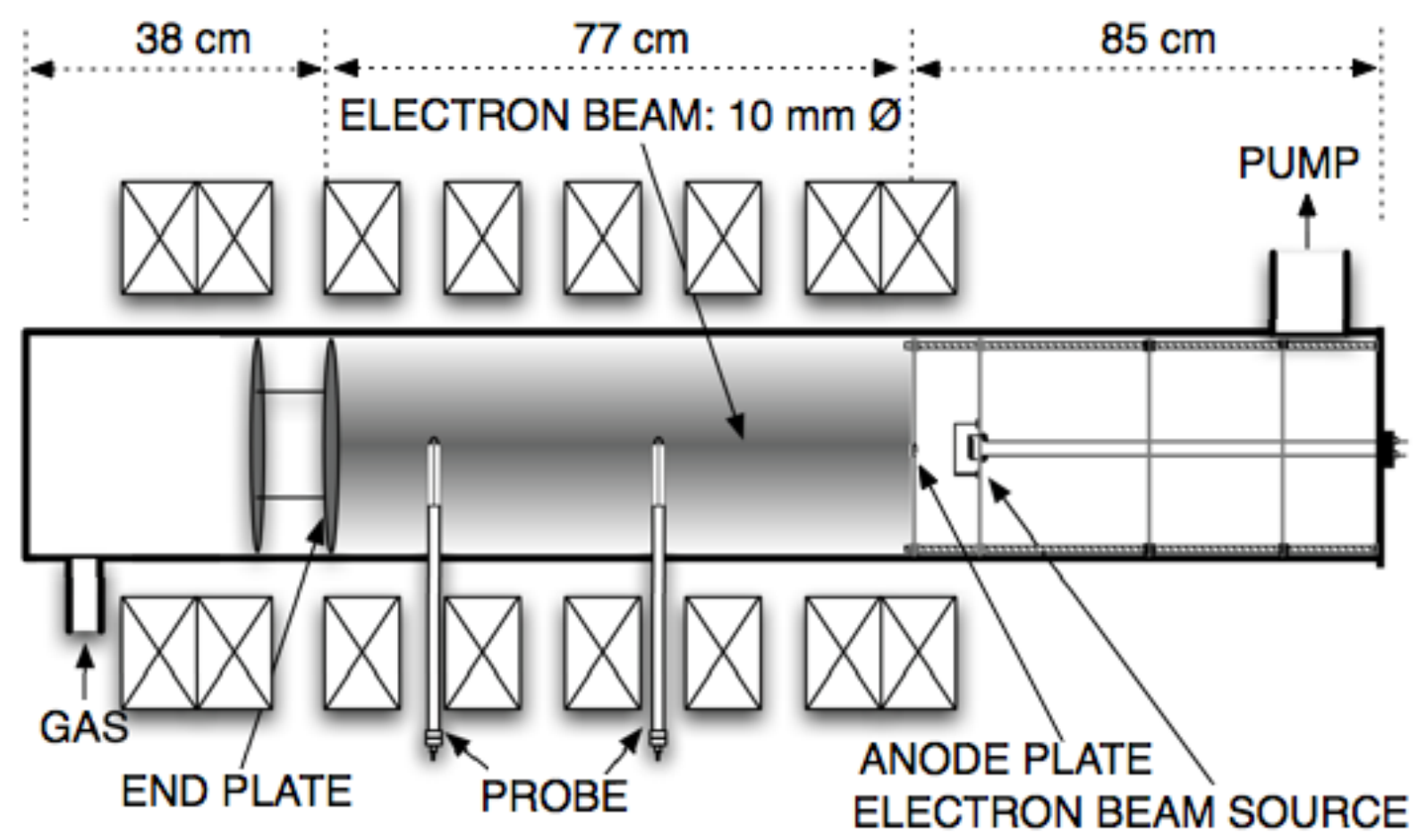

Fig. 2. Illustration of experimental setup for string-type plasma. 


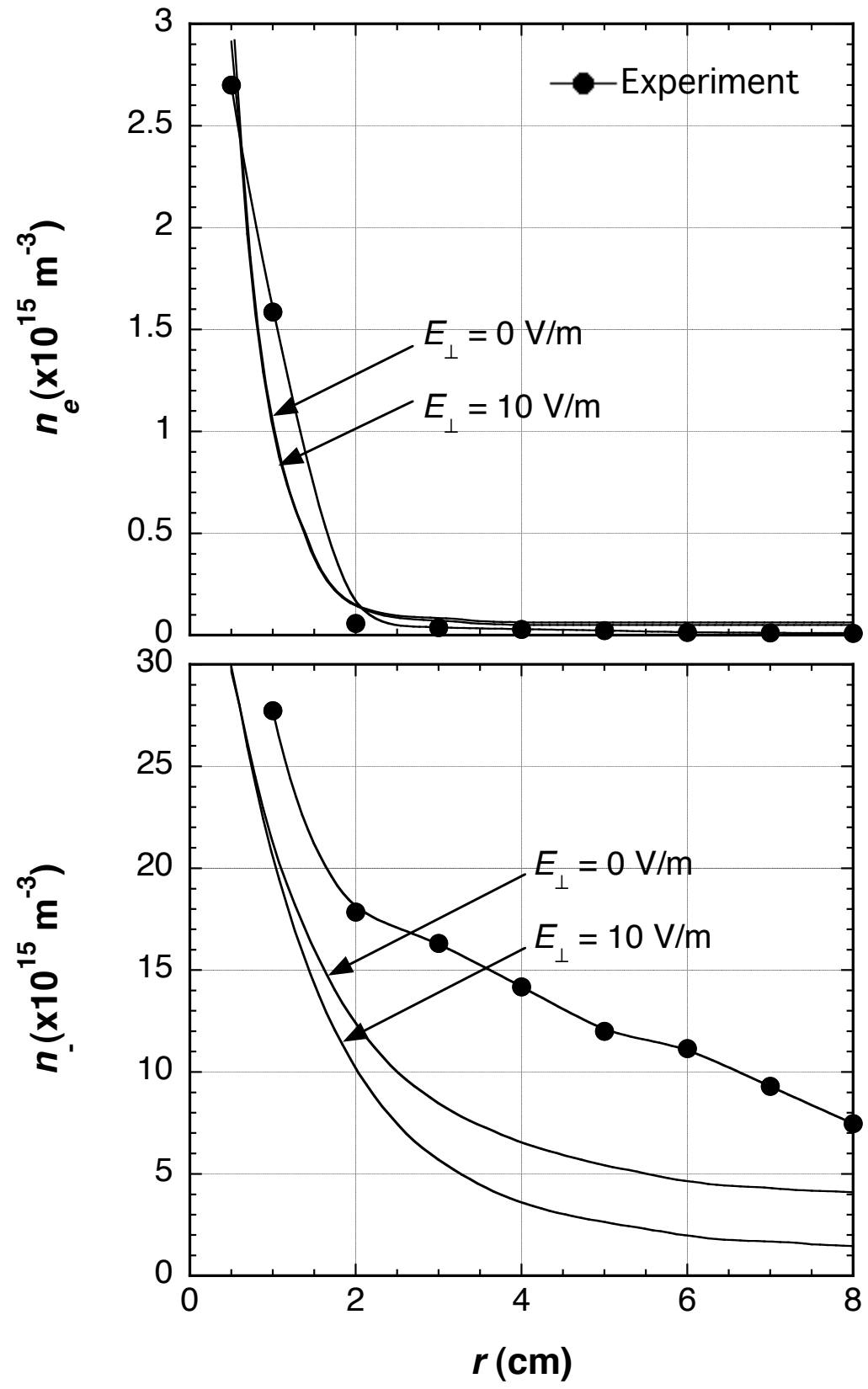

(a)

(b)

Fig. 3. Radial profiles of (a) $n_{e}$ and (b) $n_{-}$obtained by experiment and using model calculations. $\quad D_{\perp e}=0.04 \mathrm{~m}^{2} \mathrm{~s}^{-1}$ and $\mu_{\perp e}=0.14 \mathrm{~m}^{2} \mathrm{~V}^{-1} \mathrm{~s}^{-1} . \quad D_{\perp-}=4.7 \mathrm{~m}^{2} \mathrm{~s}^{-1}$ and $\mu_{\perp e}=38.5$ $\mathrm{m}^{2} \mathrm{~V}^{-1} \mathrm{~s}^{-1} . \quad B=0.03 \mathrm{~T}$ and $p=0.6 \mathrm{~Pa}$ are measured at $z=21 \mathrm{~cm}$ from the anode. 


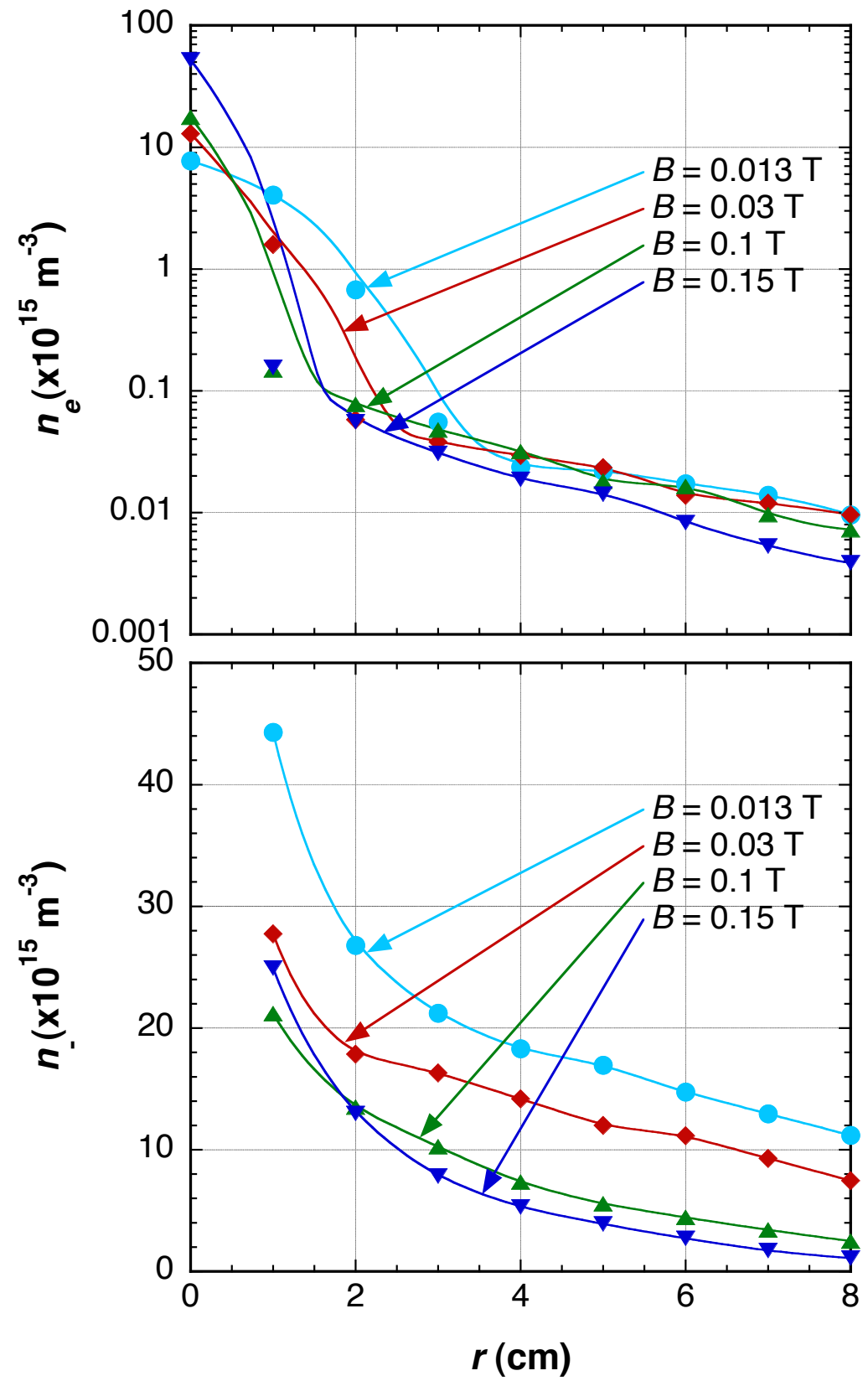

(a)

(b)

Fig. 4. Radial profiles of (a) $n_{e}$ and (b) $n_{-}$for four magnetic fields obtained experimentally at a pressure of $p=0.6 \mathrm{~Pa} . z=21 \mathrm{~cm}$ from the anode. 


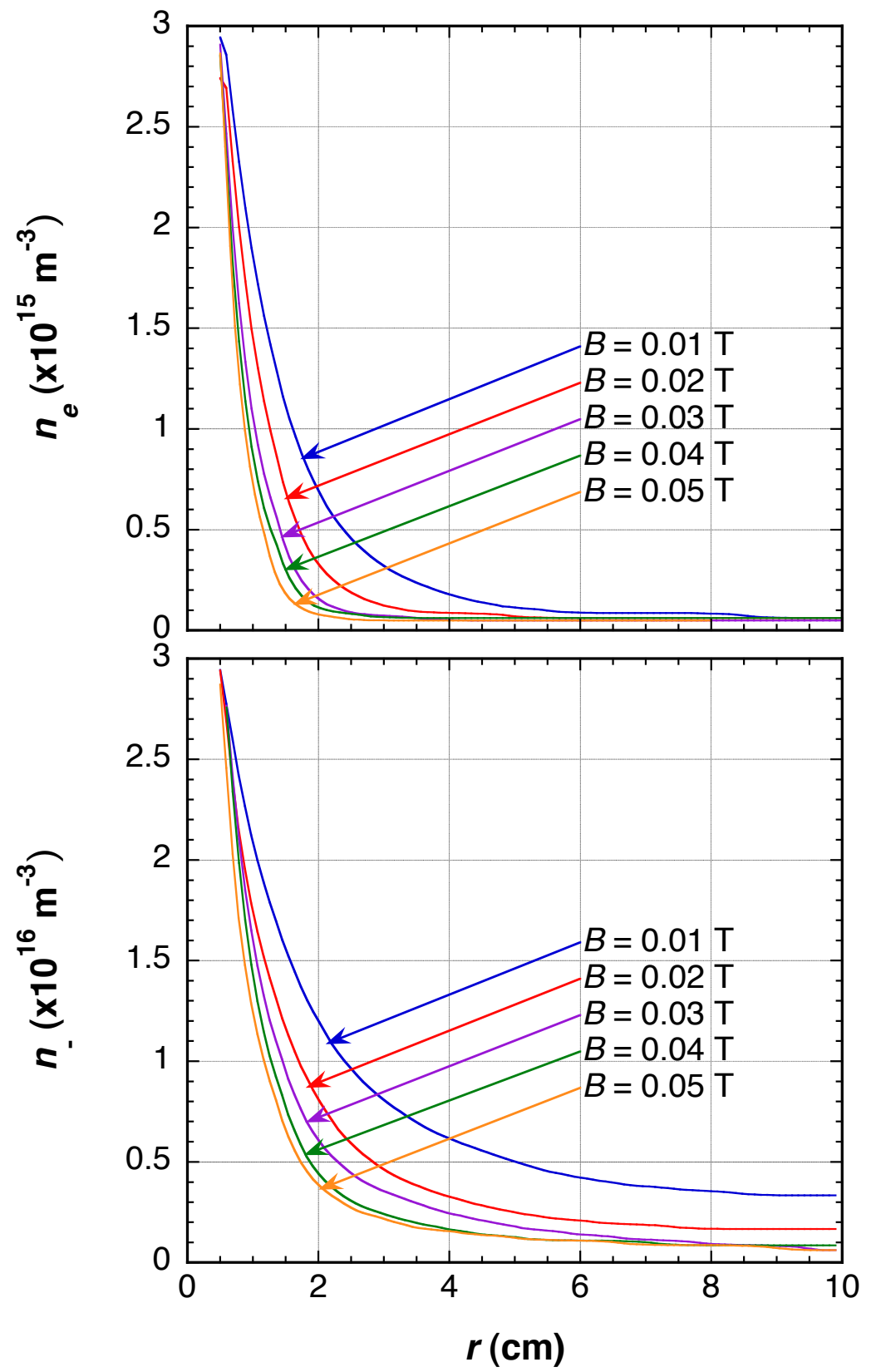

(a)

(b)

Fig. 5. Magnetic field dependences of radial profiles of (a) $n_{e}$ and (b) $n_{-}$calculated using the model equations. $\quad p=0.6 \mathrm{~Pa}$. 


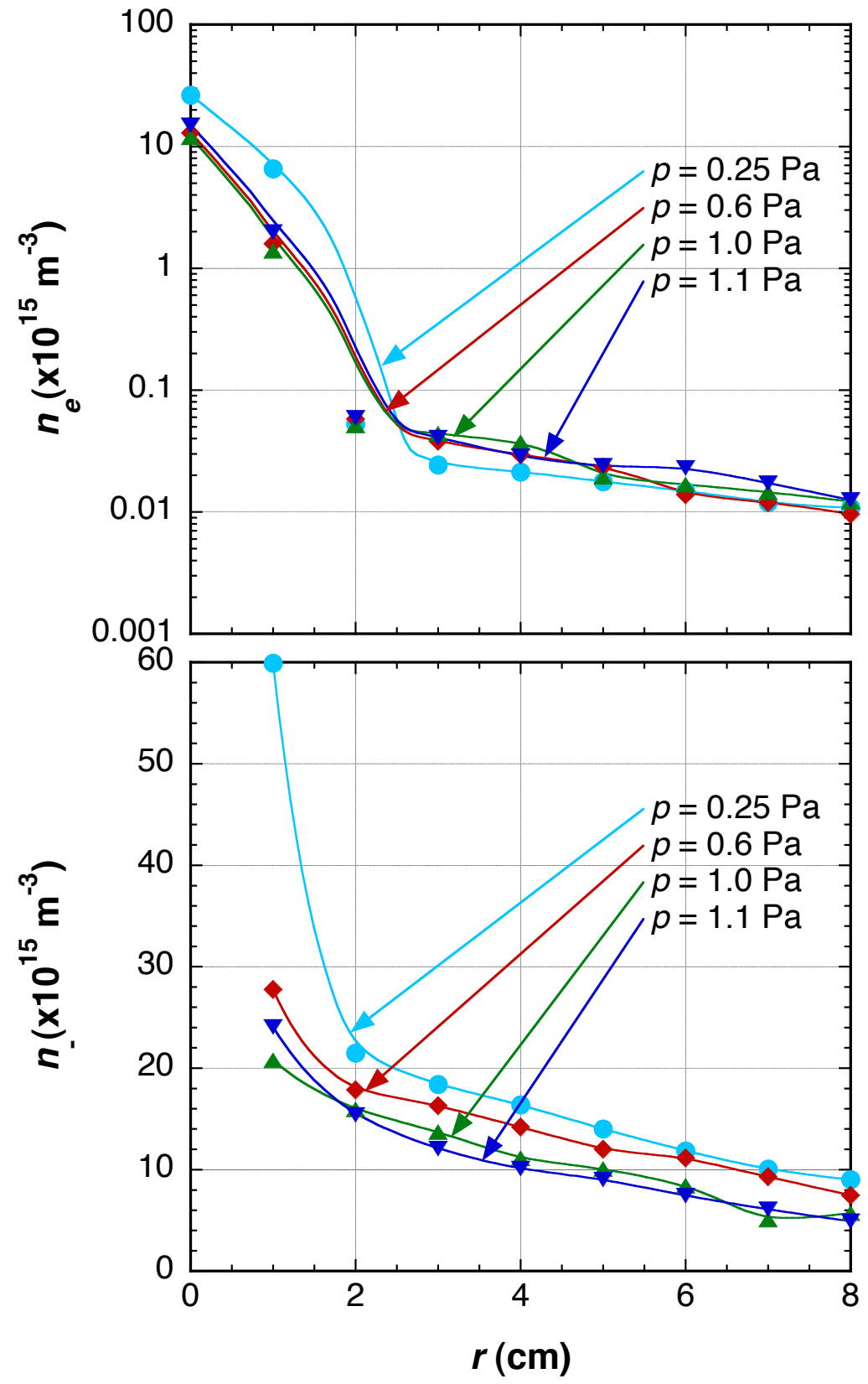

(a)

(b)

Fig. 6. Radial profiles of (a) $n_{e}$ and (b) $n_{-}$for four gas pressures obtained experimentally in a magnetic field of $B=0.03 \mathrm{~T} . \quad z=21 \mathrm{~cm}$ from the anode. 


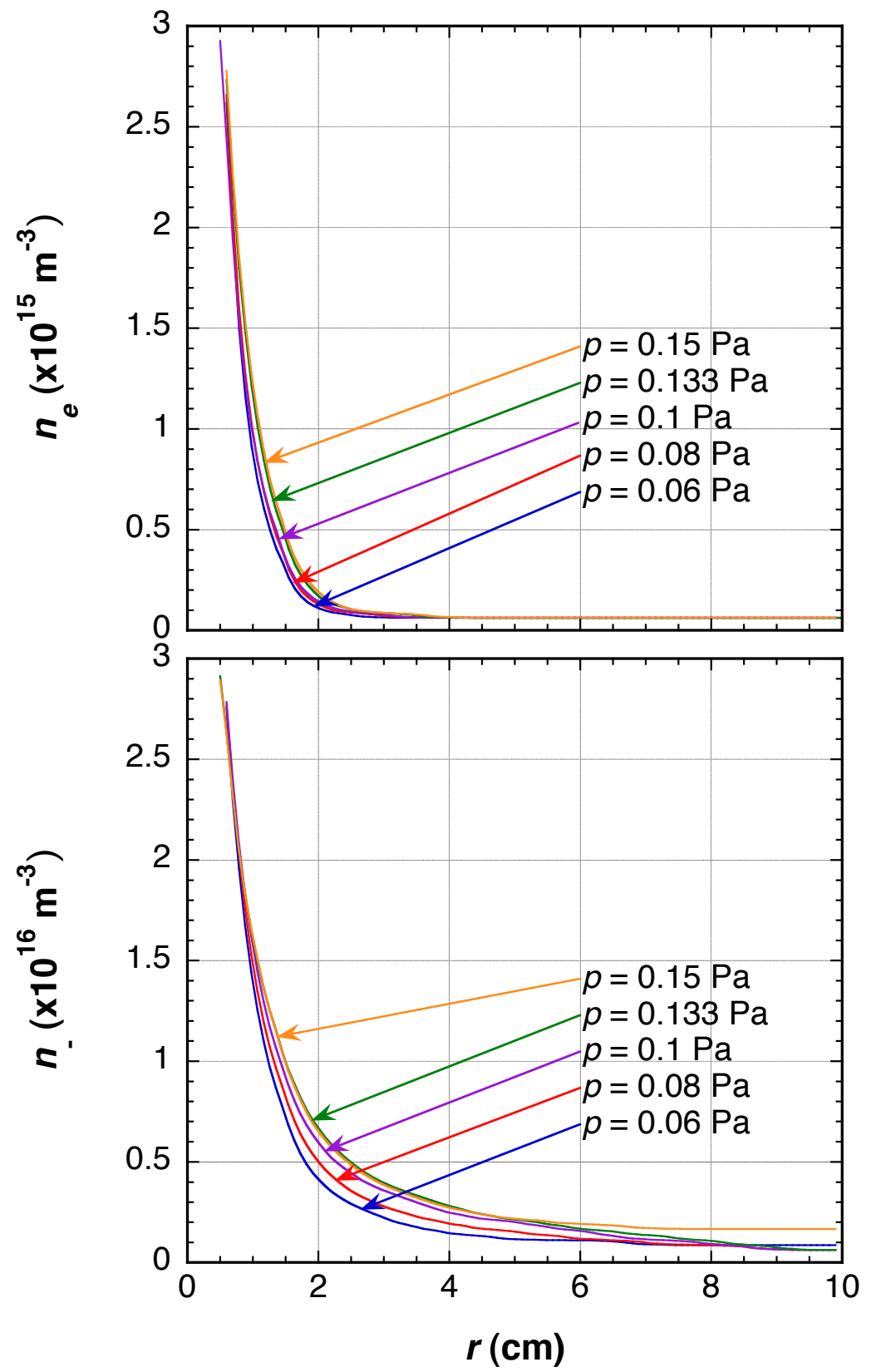

(a)

(b)

Fig. 7. Dependence of radial profiles of (a) $n_{e}$ and (b) $n_{-}$on gas pressure calculated using the model equations. $\quad B=0.03 \mathrm{~T}$. 


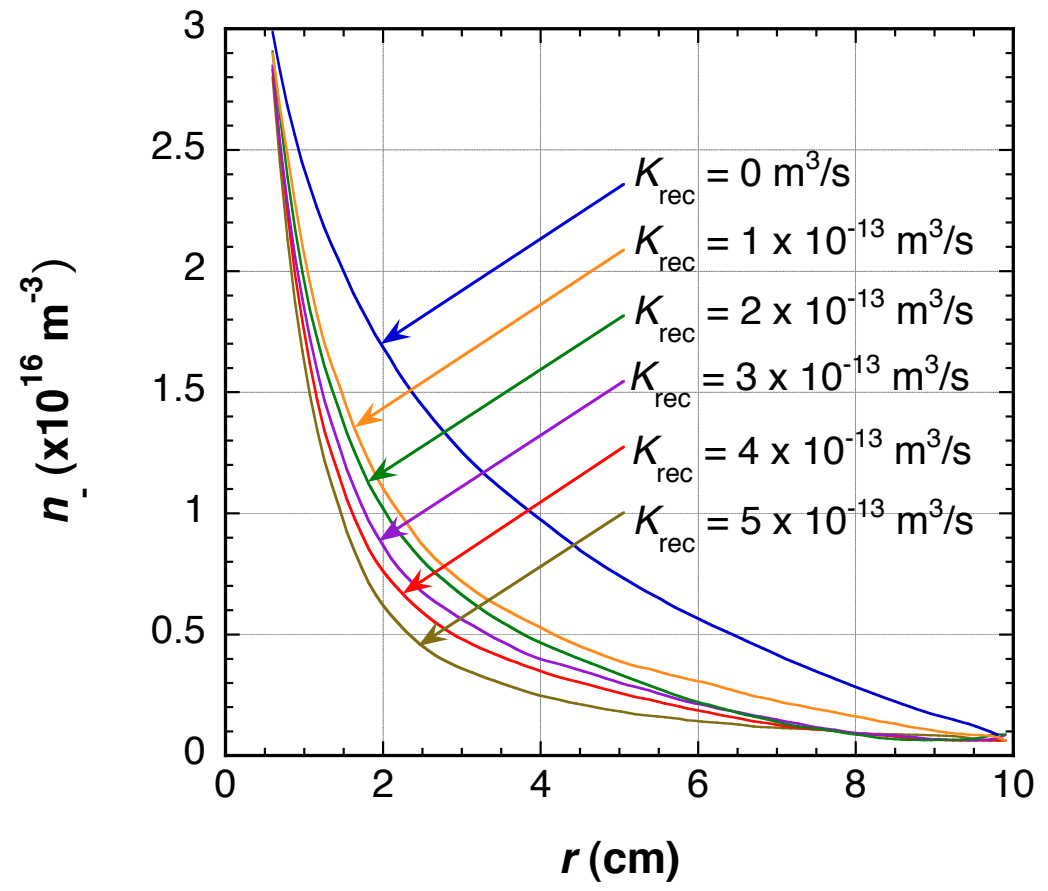

Fig. 8. Dependence of radial profiles of (a) $n_{-}$and (b) $n_{+}$on recombination coefficient calculated using the model equations. $D_{\perp_{-}}=0.5 \mathrm{~m}^{2} \mathrm{~s}^{-1}$. 


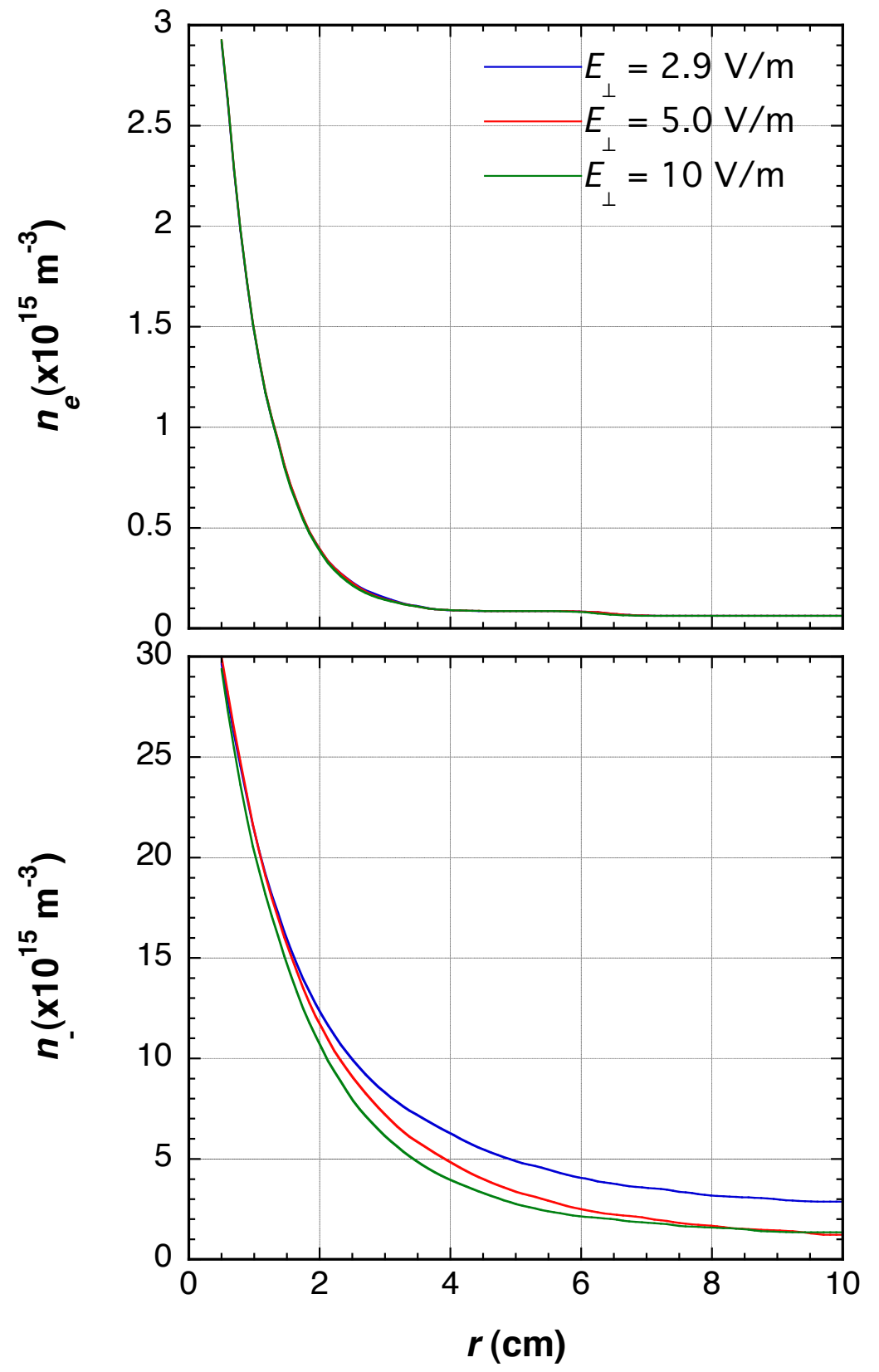

(a)

(b)

Fig. 9. Radial profiles of (a) $n_{e}$ and (b) $n_{-}$calculated using the model equations for $E_{\perp}=2.9,5.0$, and $10 \mathrm{Vm}^{-1} . \quad B=0.03 \mathrm{~T}, T_{e}=1.0 \mathrm{eV}$ and $T_{-}=0.1 \mathrm{eV}$. 